\title{
Traffic Load-Based Spectrum Trading between Two Mobile Network Operators in HetNets Using Interference Management Technique
}

\author{
Hafiza Ammara Khurshid ${ }^{D},{ }^{1}$ Farrukh Aziz Bhatti, ${ }^{1}$ Kashif Mehmood, ${ }^{2}$ Sobia Jangsher, \\ and Aamir Habib ${ }^{1}$ \\ ${ }^{1}$ WiSP Lab, Department of Electrical Engineering, Institute of Space Technology, Islamabad, Pakistan \\ ${ }^{2}$ Bahria University Karachi, Karachi, Pakistan \\ Correspondence should be addressed to Hafiza Ammara Khurshid; ammara09es24@gmail.com
}

Received 20 August 2021; Accepted 22 December 2021; Published 28 February 2022

Academic Editor: L. J. García Villalba

Copyright (c) 2022 Hafiza Ammara Khurshid et al. This is an open access article distributed under the Creative Commons Attribution License, which permits unrestricted use, distribution, and reproduction in any medium, provided the original work is properly cited.

\begin{abstract}
The pursuit of achieving higher data rates in $5 \mathrm{G}$ and beyond has triggered numerous technological advancements. One of these is spectrum sharing between Mobile Network Operators (MNOs). In this work, we investigate the spectrum trading between two MNOs with disparate traffic loads in heterogeneous networks. A radio resource sharing technique is proposed that exploits resource block allocation with Interference Range (RBIR) to reduce interoperator interference prompted by spectrum trading. In addition, an algorithm for cooperative load-based spectrum trading at resource block level is proposed for two MNOs with asymmetric traffic load. Two main approaches for spectrum trading, i.e., spectrum sharing (Load-Based Shared Spectrum Pool (LSSP)) and spectrum leasing (Load-Based Leased Spectrum Pool (LLSP)) are analysed, and a heterogeneous network is modeled such that both operators have a dedicated spectrum for their macro and small cell networks. Prior to spectrum trading, a conventional resource block allocation technique is used in which resource blocks from a shared or leased spectrum pool are assigned so that each user experiences the least amount of interference. The performance of LSSP and LLSP is evaluated while taking into account the constraints of both operators, such as net throughput and user satisfaction. Moreover, we evaluate the network performance with increasing interference range as small cells of different transmission/interference range that are present in the network. The performance evaluation demonstrates that when the interference is lesser, as represented by the radius of the interference range, the spectrum sharing is more advantageous in terms of delivering higher average data rates. However, when the interference is higher, then leasing is more beneficial.
\end{abstract}

\section{Introduction}

The evolution of wireless communication technology towards $5 \mathrm{G}$ and beyond comes with increased data rate, capacity, coverage, throughput, high mobility, and low latency. The goal of the $5 \mathrm{G}$ wireless operators is to connect around a hundred billion devices, which could not be achieved by simple enhancement and improvement in the previous generations [1]. With this tremendous increase in data rate demand, spectrum scarcity is a real challenge for wireless operators. To address the spectrum concerns, the solution is the licensed spectrum sharing among cellular operators, also termed as interoperator spectrum sharing [2], whereas other solutions such as unlicensed spectrum sharing like $5 \mathrm{GHz}$, cognitive radio networks, joint radar and communication, and deployment of heterogeneous networks have been explored in literature.

Throughout the years, many physical and network layer solutions have been proposed for better spectrum utilization. One of the key enablers for improving spectrum utilization is the use of small cells, which is known as heterogeneous networks (HetNets). The small cells increase the frequency reuse factor by allowing more spectrum to be allocated per unit area. Small cells encompass low-powered base stations 
that include femtocells, picocells, and microcells with varying coverage regions [3].

In addition to that, sharing resources between two or more Mobile Network Operators (MNOs) is a promising solution that offers great advantages. With any type of sharing, it is important for MNOs to know the achieved capacity gains. For that matter, two types of sharing exist in the literature: (a) an active sharing and (b) passive sharing. According to Frisanco et al. [4], active sharing includes cell site sharing, infrastructure sharing, and equipment sharing, whereas passive sharing involves the sharing of the radio spectrum. In Ref. [5], a detailed study is performed on physical resource sharing (spectrum) and infrastructure sharing individually and collectively. The results showed significant gains in the overall network performance as well as enhanced economic benefits [5]. Spectrum sharing between MNOs with small cell network (SCNs) deployment can increase their spectrum efficiency without increasing the bandwidth and system deployment costs. However, spectrum sharing in HetNets needs to be handled meticulously; otherwise, it can cause interference which can undermine the potential benefits. The spectrum sharing problem is solved by taking into account different scenarios; the difference lies in the way interoperator interference is catered, i.e., through the separation of signals in either time, frequency, or space [6,7]. In Ref. [8], the authors investigate the wireless access to the shared bands and the conclusion states that the proposed solution is much more efficient than the use of a dedicated spectrum by an operator. Similarly, in Ref. [9], multi-operator dynamic frequency selection (DFS) approach with spectrum sharing provides better performance compared to no sharing. Cooperative spectrum sharing is considered an effective way to enhance spectrum efficiency, which in turn results in capacity increase [10]. Yang et al. in Ref. [11] use different terminologies for spectrum sharing with respect to technical and economic perspectives, such as spectrum trading, spectrum leasing, spectrum sharing, spectrum mobility, spectrum routing, and spectrum harvesting. In this work, we refer to the aforementioned first three terminologies. Spectrum trading implies that the licensed operators trade their exclusively assigned spectrum with other parties that could be other licensed operators or unlicensed operators such as cognitive radio. Spectrum trading can be done in different ways, including leasing or sharing. Moreover, when an operator offers its spectrum to another operator or service for exclusive usage, then this is referred to as spectrum leasing. However, when the same is offered to another operator or service on a sharing basis, then this is referred to as spectrum sharing. Both sharing and leasing have their pros and cons; therefore, choosing the right technique in a particular traffic load condition involving multiple operators is crucial in maximizing the overall spectrum efficiency. It is particularly effective in the case of fluctuating spectrum demand over time. In our case, we are dealing with two operators only.

Pricing strategies are well-known strategies that have been studied for sharing scenarios [12-14]. The ethical and legal issue related to identity privacy and data security is one of the major concerns for secure spectrum sharing among multiple operators. To address this challenge, Zheng et al. propose a smart contract with the constructed permissioned block-chain trust framework for spectrum sharing in multiple wireless networks [15]. However, security and pricing strategies are beyond the scope of our paper. Interference management in HetNets is a challenging task with many degrees of assignment in detail and spectrum trading adds to its complexity; thus, it needs to be handled intelligently. The increase in the number of users associated to a specific cell causes more interference to the co-channel cells and degrades the signal to interference plus noise ratio (SINR). The fact that cell load is contingent on SINR creates an inherent contradiction between capacity and load and leads to an inefficient resource allocation [16,17]. The objective of this paper is to present an efficient resource allocation mechanism for shared spectrum based on load disparity which minimizes the resulting interference. The main contributions of this paper are as follows:

(i) We propose an efficient and effective load-based spectrum trading (i.e., LSSP and LLSP) scheme for densely deployed outdoor small cell networks of two operators

(ii) We analyze the proposed scheme in two different scenarios which depends on the load disparity of the two operators such that each user experiences the best SINR

(iii) Furthermore, we investigate an effective resource allocation technique with minimal interference (i.e., Resource Block allocation with Interference Range (RBIR)) for both scenarios

Based on our simulation results, we conclude the most efficient and effective scheme (spectrum leasing or sharing) for each of the considered scenarios. The paper is structured as follows. Section 2 explains the literature related to spectrum sharing and leasing in detail. The system model is described in Section 3. Section 4 analyzes the proposed algorithm for load-based spectrum trading for both considered scenarios, along with the interference management techniques. The system-level performance is evaluated and discussed in Section 5. Finally, the conclusion and future recommendations are explained in Section 6.

\section{Related Work}

Heterogeneous networks of different mobile network operators sharing spectrum in the same geographical region can exploit the variable load index (a quantified measure of traffic load) and accomplish mutual benefits by managing the allocation of resource blocks (RBs). Tehrani et al. presented a study in Ref. [18] where the concept of dynamic spectrum sharing was described by taking different scenarios into consideration and highlights the major challenges of spectrum sharing. In Refs. [19-21], the authors presented a co-primary spectrum sharing (CoPSS) based on ask and grant of spectrum usage favor using repeated game theory for indoor HetNets. In addition, Singh et al. in Ref. [20] proposed solutions where the operators generate a limited 
spectrum pool which can be used in a variety of ways depending on the interference conditions. Both operators attain benefit from spectrum coordination for imbalanced load. Moreover, noncooperative repeated games were used to explore spectrum sharing between two SCN operators; however, in their case, the operators share a set of component carriers from a shared spectrum pool [20].

Aside from that, the authors of Ref. [19] provided a spectrum sharing utilizing repeated games through game theory and concealing operator-specific information. When the network load varied over time, the proposed technique outperforms the static spectrum sharing rules. Furthermore, in Ref. [21], repeated game theory was applied to a scenario in which many operators collaborated to build a pool in the downlink between microcell and femtocell networks. The indoor femtocell network shares spectrum to provide improved coverage to the other operator's outdoor microcell users that are perceived as visiting user equipment.

Luoto et al. in Ref. [22] presented CoPSS in terms of centralized and decentralized sharing algorithms at the physical resource block level that cause higher spectral efficiency, whereas CoPSS refers to orthogonal sharing, which reduces the interference and maximizes bandwidth utilization. In another work, Luoto et al. [23] extended their work with enhanced CoPSS and included a fairness index between sharing algorithms that compute for an indoor small cell deployment. Moreover, they compared various state-of-the-art (no sharing, equal sharing, and greedy) techniques with transcendentalist algorithms (Gibbs, penalty, and distribution) [23].

In Ref. [24], Jorswieck et al. investigated the potential gain of spectrum sharing between MNOs in terms of network efficiency, in which they considered four cases of spectrum sharing: (a) intra-operator spectrum sharing, (b) interoperator orthogonal spectrum sharing, (c) interoperator nonorthogonal spectrum sharing, and (d) no spectrum sharing. However, the limitation of the presented solutions was that they did not explicitly identify network conditions in which either of the spectrum sharing performs better or worse. This limitation is addressed in our work where we identify the network conditions, i.e., the size of the small cell and the number of users within (load traffic on each small cell) that are more suitable for either spectrum leasing or sharing.

In Ref. [25], the authors proposed a game theoretical framework for multiple MNOs to share a common pool of spectrum among users. Their objective was to maximize a social welfare index, which can be defined as the overall weighted sum rate of the operators. Furthermore, interference was managed by allocating RBs from multiple MNOs to users based on many-to-one matching game with externalities. However, they considered network social characteristics as the criteria for spectrum sharing instead of traffic load requirements.

For interoperator spectrum sharing, a joint common property rights model was proposed in Ref. [26], where one operator offloads its macrocell network traffic to the other operator's SCN. In addition to that, Kibilda et al. in Ref. [27] presented a study of radio access network (RAN) sharing along with spectrum sharing among different MNOs and the effect of spectrum sharing on RAN was analysed. In Ref. [28], Gangula et al. demonstrated an adaptive spectrum sharing policy based on user position and small cell position distribution. Regarding that, the user's position in the macro and small cell determines the allocation of orthogonal (unshared) and nonorthogonal (shared) resource blocks between MNOs. Nonorthogonal resource block allocation resulted in interference among MNOs, whereas orthogonal resource block allocation avoided interference [28]. In Ref. [29], Anand et al. investigated a price and bandwidth management approach using game theory. Pertaining to that, two mechanisms, namely, uniform pricing and proportional bandwidth advertisement were proposed. Moreover, the authors also presented a bandwidth allocation scheme in cognitive HetNets to provide the best quality of service while maximizing the revenue of the wireless service provider [29].

Saha in Ref. [30] discussed a number of spectrum (licensed and unlicensed) sharing techniques for in-building small cells, namely, dedicated access, co-primary shared access (CoPSA), licensed shared access (LSA) for licensed spectrum access, and licensed assisted access (LAA) for unlicensed spectrum operating in $60 \mathrm{GHz}$. In this context, the almost blank subframe (ABS)-based eICIC technique has been used to avoid or minimize co-channel interference signals generated due to sharing the same spectrum in space simultaneously. The small cells are facilitated with dual-band and derive the average capacity, spectral efficiency, and energy efficiency metrics for each technique. In Ref. [31], the authors proffered a dynamic exclusive-use spectrum access (DESA) method for indoor small cells to improve the overall licensed millimeter wave (mmWave) spectrum utilization for an arbitrary number of MNOs in a country. An iterative algorithm was proposed to find the optimal amount of shared spectrum for each MNO, and was updated at each round of negotiation; however, the performance evaluation was made in terms of average capacity, spectral efficiency, energy efficiency, and cost efficiency.

Furthermore, Kotagi et al. [2] illustrated the importance of interoperator proximal spectrum sharing by theoretically analysing the handover probability, coverage probability, and achieved data rate. Regarding that, the authors proposed an energy efficient Q-Learning-based framework which has minimized the energy consumption of the system without trading off throughput and addressed the interference problem to the cell edge users by applying it in a proximal spectrum sharing scenario. In addition, a hybrid modelbased and data-driven multi-operator spectrum sharing mechanism was proposed in Ref. [32]. The technique incorporates model-based beam forming and user association complemented by data-driven model refinements having substantially less signalling overhead but at the expense of increased interoperator interference. In Ref. [33], AlAmmouri et al. proposed a load aware model for per-user power control with limited transmit power for uplink cellular network in multichannel environment such that each user inverts its pathloss to a limited transmit power. The proposed scheme not only does the proposed power control but 
also provide balanced cell center and cell edge user performance. Contrary to this work, we proposed multi-operator spectrum sharing based on load for the downlink cellular network to enhance the cell edge user throughput.

Based on the described literature, our investigation is "standing on the shoulders of others." The discussed techniques, from researchers around the world, guide us to propose the best possible solutions for the investigated problems which address the limitations found in literature.

\section{System Model}

We consider two HetNet MNOs, i.e., operator $A$ and operator $B$. Both MNOs have co-located macro base stations (MBSs) with similar coverage area, as shown in Figure 1. Each operator has a dedicated licensed spectrum, which is distributed among macro and small cell networks. Pertaining to that, the bandwidth is subdivided into smaller units of $q \mathrm{~Hz}$ that spans over a time duration of $w$ seconds, known as resource block. We contemplate an outdoor SCN that provides better coverage and capacity to the users in general, especially at the edges of the macrocell. We denote the set of all small cells of an operator as $m=\left\{1,2,3, \ldots, M_{O}\right\}$ and the total number of RBs as $k=\left\{1,2,3, \ldots, K_{O}\right\}$, where $M_{O}$ is the total number of small cells and $K_{O}$ is the total number of RBs in $O: O \in A, B$. All the parameters and their descriptions are listed in Table 1.

Let us assume that the MNOs are aware of each other's traffic load, where $L_{O}$ is the traffic load of each operator $O: O \in\{A, B\}$. The load of an operator is defined as:

$$
L_{O}=\sum_{n=1}^{N_{O}} \alpha_{n}
$$

where $\alpha_{n}$ is the required capacity of the user $n$ (the user can be a macrocell or a small cell user) of MNO $O$. The total number of users served by each operator is given as

$$
N_{O}=N_{O}^{\prime}+N_{O}^{\prime \prime}
$$

where $N_{O}^{\prime}$ and $N_{O}^{\prime \prime}$ represent the total number of macrocell and small cell users of MNO O. Without loss of generality, we consider two operators with asymmetric load, i.e., $L_{A}>L_{B}$. This assumption is based on certain studies presented in Refs. [34,35] that have shown significant fluctuations in network traffic load over temporal and spatial domains. Consequently, the operators can mutually benefit from these independent fluctuations in traffic load and can do spectrum trading accordingly.

The SINR, $\gamma_{n^{\prime \prime}, k}$ experienced by the users on the downlink for spectrum trading is expressed as

$$
\gamma_{n^{\prime \prime}, k}=\frac{P_{n^{\prime \prime}, k}}{n_{o}+I_{O, n^{\prime \prime}, k}+I_{O^{\prime}, n^{\prime \prime}, k}},
$$

where $P_{n^{\prime \prime}, k}$ is the received signal power on the downlink, $n_{o}$ is the noise power, $I_{O, n^{\prime \prime}, k}$ is the interference experienced by the operator's own small cell base stations (SBSs), and $I_{O^{\prime}, n^{\prime \prime}, k}$ is the interference generated by the SBSs of other network in Shared Spectrum Pool (SSP) and this term is 0 for the case of
Leased Spectrum Pool (LSP). The downlink transmission data rate of user $n^{\prime \prime}$ associated with small cell $m$ on RB $k$ is given as

$$
R_{n^{\prime \prime}, k}^{m}=w_{n^{\prime \prime}, k} B_{k} \log _{2}\left(1+\gamma_{n^{\prime \prime}, k}\right) .
$$

Proportionally fair scheduling $w_{n^{\prime \prime}, k}$ is performed for all users. $B_{k}$ is the size of RB. The achievable data rate for the SCN for spectrum trading is expressed as:

$$
U_{O, C}=\sum_{m=1}^{M_{O}} \sum_{n^{\prime \prime}=1}^{N_{O}^{\prime}} \sum_{k=1}^{K_{T}}\left(R_{n^{\prime \prime}, k}^{m}\right),
$$

where $K_{T}=K_{s} \cup K_{O}$ for SSP, $K_{T}=K_{A}+K_{L}$ for operator $A$ in LSP, and $K_{T}=K_{B}-K_{L}$ for operator $B$ in LSP. To achieve a higher throughput, SINR should be high as well, conversely, lower SINR results in lower throughput. In the case of sharing, the users experience higher interference, due to additional interference from the other operator sharing the resources. However, while using the operator's own resources only, there is no interference from the other operator, consequently there is lesser interference overall. This can be observed in (3), where the only interference present is from the low powered SBSs of the same operator. To represent this interference, we assume a protocol interference model [36], which is discussed in Section 4. Our goal is to evaluate the overall throughput of $\mathrm{MNO}$, and to evaluate how much an operator gains from cooperative spectrum sharing by computing the difference of throughput with and without cooperation. Mathematically, we observe that without sharing there will be no interference by the collaborator (operator $O^{\prime}$ ) i.e., the factor $I_{O^{\prime}, n^{\prime \prime}, k}=0$. The achievable data rate without sharing/leasing is given as

$$
U_{O, O}=\sum_{m=1}^{M_{O}} \sum_{n^{\prime \prime}=1}^{N_{O}^{\prime}} \sum_{k=1}^{K_{O}}\left(R_{n^{\prime \prime}, k}^{m}\right) .
$$

Thus, the overall gain achieved through sharing/leasing is expressed as

$$
G_{O}=U_{O, C}-U_{O, O}
$$

\section{Load-Based Spectrum Trading}

In this section, we propose a centralized Load-Based Spectrum Trading (LBST) algorithm to utilize the resources efficiently in an MNO. The operators share their load index with the central controller, which in turn pairs the two operators and decides a trading strategy. The central controller has two different trading strategies: (1) Sharing and (2) Leasing. Based on the load index variation between the operators, the central controller decides on the best spectrum trading strategy.

We consider an outdoor scenario for a SCN with a random, dense deployment of SBSs. It should be noted that the SBSs are not deployed near MBS. Prior to requesting spectrum trading, we use a conventional resource allocation scheme to allocate RBs to the macro and small cell network users based on SINR [37]. As described earlier, we have 


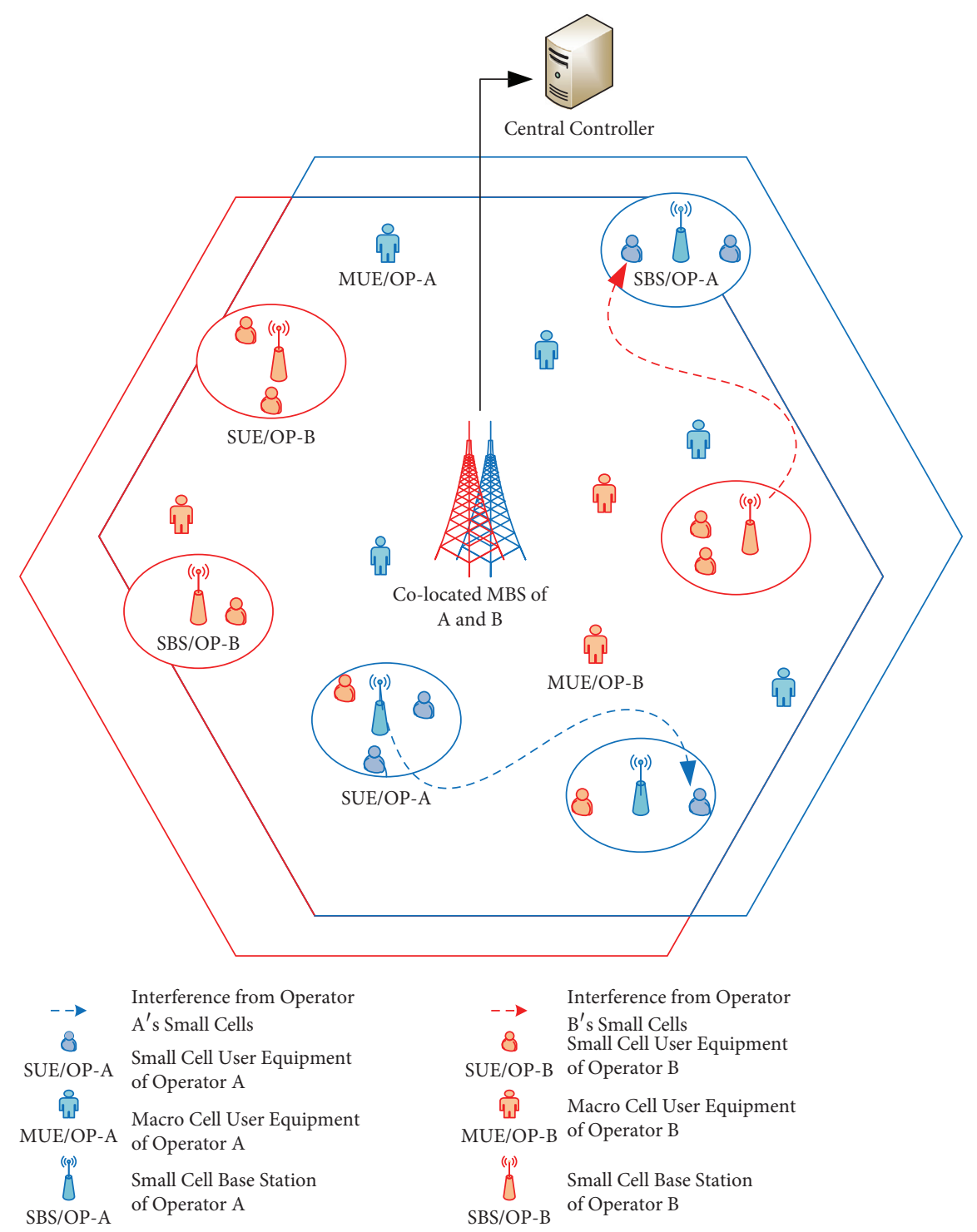

Figure 1: Two operator HetNet deployment scenario.

TABle 1: Model parameters and description.

\begin{tabular}{lc}
\hline Parameters & Description \\
\hline$O$ & Set of mobile network operators \\
$L_{O}$ & Total load on each MNO \\
$N_{\rho}^{\prime}$ & Number of macrocell users of each MNO \\
$N_{O}$ & Number of small cell users of each MNO \\
$M_{O}$ & Number of small cells of each MNO \\
$K_{T}$ & Total number of resource blocks \\
$K_{S}$ & Number of shared resource blocks \\
$K_{L}$ & Number of leased resource blocks \\
$K_{O}$ & Number of MNO's own resource blocks \\
$D_{O, N}^{M}$ & Set of requested data rate by users \\
$\mathbf{Q}_{\mathrm{O}, \mathrm{N}}^{M}$ & Set of users not getting requested data rates \\
$\mathbf{Z}$ & Total number of disk zones \\
$\mathscr{M}$ & Set of RBIRs for each zone \\
\hline
\end{tabular}

dedicated bandwidth for macro and small cell networks. Here, in conventional RB allocation, we serve the users based on the best SINR conditions. For each user, either a macrocell user or a small cell user SINR is calculated. No inter-cell interference is present as dedicated spectrum allocation is considered between macrocells and small cells. The achievable data rate requirements of macrocell and small cell users are represented as $D_{O, n^{\prime}}$ and $D_{O, n^{\prime \prime}}^{m}$, respectively. The average number of RBs for each macrocell and small cell can be determined as.

$$
\begin{aligned}
& \lambda_{O, n^{\prime}}=\frac{D_{O, n^{\prime}}}{\Gamma_{k}}, \\
& \mu_{O, n^{\prime \prime}}^{m}=\frac{D_{O, n^{\prime \prime}}^{m}}{\Gamma_{k}} .
\end{aligned}
$$

respectively. Here $\Gamma_{k}$ is the average achievable data rate per RB.

Algorithm 1 shows a conventional RB allocation scheme for macro and small cell users. Let $R_{\mathrm{O}, \mathrm{n}^{\prime \prime}}^{\mathrm{m}}$ be the data rate 
calculated for each SCN user with required number of RBs from selected SBS, and $Q_{\mathrm{O}, \mathrm{N}^{\prime \prime}}^{\mathrm{m}}$ are the users of operator $O$ and small cell $m \in M_{O}$ not getting their requested data rates. To overcome capacity deficiency, an operator needs more spectrum.

Mutual spectrum usage among multiple operators leads to a new paradigm. As discussed in Sections 1 and 2, different spectrum trading strategies have been implemented in literature. However, we consider and explain two scenarios of LBST, i.e., the operator either leases its spectrum or shares it.

(i) Scenario I: Shared Spectrum Pool (SSP) which is used by both MNOs and is referred to as Load-Based Shared Spectrum Pool (LSSP).

(ii) Scenario II: Leased Spectrum Pool (LSP) which is used exclusively by the MNO who requests for the additional spectrum and is referred to as Load-Based Leased Spectrum Pool (LLSP).

We consider the terms and conditions for spectrum trading that both operators agree upon remain the same in both cases. Next, the throughput achieved by the two MNOs for the aforementioned scenarios is discussed. The proposed LBST algorithm for RB allocation is based on the mutual concessions and compromises for spectrum usage, i.e., an operator requests its collaborator for pooling and both operators will start to negotiate a round of spectrum sharing cooperatively. We have made the following assumptions when the operators negotiate for LSSP or LLSP.

(i) Both operators must agree on and be aware of sharing factor $S_{O^{\prime}}$.

(ii) $T_{S S P}$ is the time span for which the operators form a shared spectrum pool. We assume that for $T_{S S P}$ the traffic conditions remain fairly constant.

(iii) Only information exchanged among operators is the time span $T_{S S P}$ and the willingness for SSP. The willingness is represented by a binary number $F$ defined as

$F= \begin{cases}1 & \text { if operator } \\ 0 & \text { otherwise }\end{cases}$

(iv) Spectrum trading (sharing or leasing) depends upon load disparity, where load disparity is defined as the ratio of the load of operator $B$ to the load of operator $A$, i.e.,

$$
\mathrm{P}=\frac{L_{B}}{L_{A}}
$$

When both operators have equal load, i.e., $\mathrm{P}=1$, then the operators neither share nor lease their spectrum. Spectrum trading only occurs when $\mathrm{P}<1$. The central controller decides to do spectrum sharing if the load disparity is above a certain set threshold $\delta$, otherwise it performs leasing. A high value of $\mathrm{P}$ indicates that the load of the two operators is comparable and in such a situation an MNO is not ready to completely give away its RBs but sharing can still enhance the data rate of the network if done carefully. Whereas a low value of load disparity implies $L_{A} \gg L_{B}$, i.e., operator $B$ has RBs in access and can lease it to the other operator. As already stated in Section $3, L_{A}>L_{B}$. The parity value $P$ is always calculated with reference to the cell with higher traffic load, i.e., $L_{A}$ is always in the denominator.

In realistic wireless networks, the network traffic load varies across space and time. In Ref. [38], the traffic load is modeled such that the variation in load does not affect the spectrum trading between MNOs. However, in this work, the load parity is assumed in the range of $0.5-1.0$. The acknowledgment time $T_{A C K}$ for SSP must be less than $T_{S S P}$, i.e., $T_{A C K} \ll T_{S S P}$. In LBST-SSP, no incentive is given to any operator. We also assume that over a fairly long time the favor sought equals the favor granted. The proposed algorithm aims to use a shared spectrum pool, i.e., the free RBs are equally assigned to the overloaded SBSs. It is assumed that sharing is performed in a distributive manner because each operator wants to maximize its own data rate.

Each operator $O$ reports its spectrum usage demand to the central controller that has the information of bandwidth utilization of each operator and it facilitates the coordination between the two operators. In a case, when the operator's load is higher than the capacity it can offer using its own allocated spectrum, then the operator is utilizing its full spectrum and needs more physical resources to fulfill its spectrum needs. Both operators cooperatively agree on spectrum trading and form a spectrum pool, as shown in Figure 2. The spectrum pool is used by the operator $O$ typically depending on the operator $O^{\prime}$. Either the operator $O^{\prime}$ forms SSP or LSP, which is coordinated via the central controller. Furthermore, in this section, we discuss both scenarios of LBST along with the interference management techniques.

4.1. Load-Based Shared Spectrum Pool (LSSP). In scenario I, the shared resources need to be allocated intelligently such that the operator sharing its resources does not suffer from performance degradation and benefits the operator receiving the favor. In spectrum pooling, the most critical issue is interference management, i.e., the operator not only experiences interference from its own small cells but also additional interference from the pooled operator's small cells. We propose Resource Block Allocation of Load-Based Shared Spectrum Pool with Interference Range (RBIR) algorithm to manage interference for a shared spectrum pool in HetNets, where both operators are using the SSP.

The main goal behind co-channel resource block allocation with RBIR is to keep the interference under control through interference management as well as resource management. We assume that the only interference is caused by the SCN. The SCNs of both operators interfere with each other as each of them shares the same spectrum pool. As explained in the system model, we have considered a protocol interference model as shown in Figure 3, which has a disk-like representation as in Ref. [36]. Each SBS has a transmission range of $r$ and its interference range is twice of its transmission range, i.e., $2 r$. If $\mathrm{RB}_{1}$ 
(1) $n^{\prime} \in N_{O}^{\prime}$ and $n^{\prime \prime} \in N_{O}^{\prime \prime}$ set of macro and small cell users

(2) User Association is performed for all $n^{\prime} \in N_{O}^{\prime}$ and $n^{\prime \prime} \in N_{O}^{\prime \prime}$ based on SINR using (3)

(3) Required number of RBs are calculated using (8) and (9) for each macro and small cell user, respectively

(4) Allocate required number of RBs to each user $n^{\prime}$ and $n^{\prime \prime}$ based on best SINR calculated in Step 2

(5) Initialize $\mathbf{Q}_{\mathrm{O}, \mathrm{N}^{\prime \prime}}^{\mathrm{M}}$ set of users whose data rate is below the threshold

(6) Calculate $R_{\mathrm{O}, \mathrm{n}^{\prime \prime}}^{\mathrm{m}}$ for each small cell user with selected SBS using (4)

(7) for $n^{\prime \prime}=1: N_{O}^{\prime}$

(8) if $R_{\mathrm{O}, \mathrm{n}^{\prime \prime}}^{\mathrm{m}} \ll d_{\mathrm{O}, n^{\prime \prime}}^{m}$ or $R_{\mathrm{O}, \mathrm{n}^{\prime \prime}}^{\mathrm{m}}=0$ store that each user $n^{\prime \prime} \in N_{O}^{\prime \prime}$ in $\mathbf{Q}_{\mathrm{O}, \mathrm{N}^{\prime \prime}}^{\mathrm{M}}$

(9) end

(10) end

(11) Repeat Step 7 for all small cells

Algorithm 1: Conventional RB Allocation.

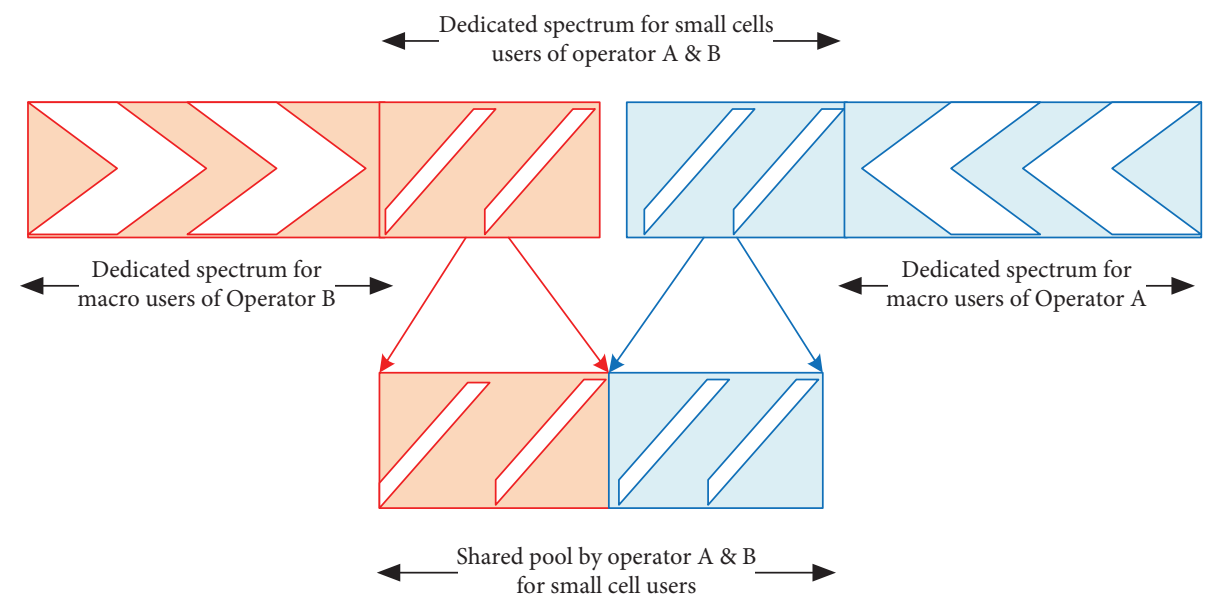

FIgURE 2: Shared spectrum pool by operators A and B

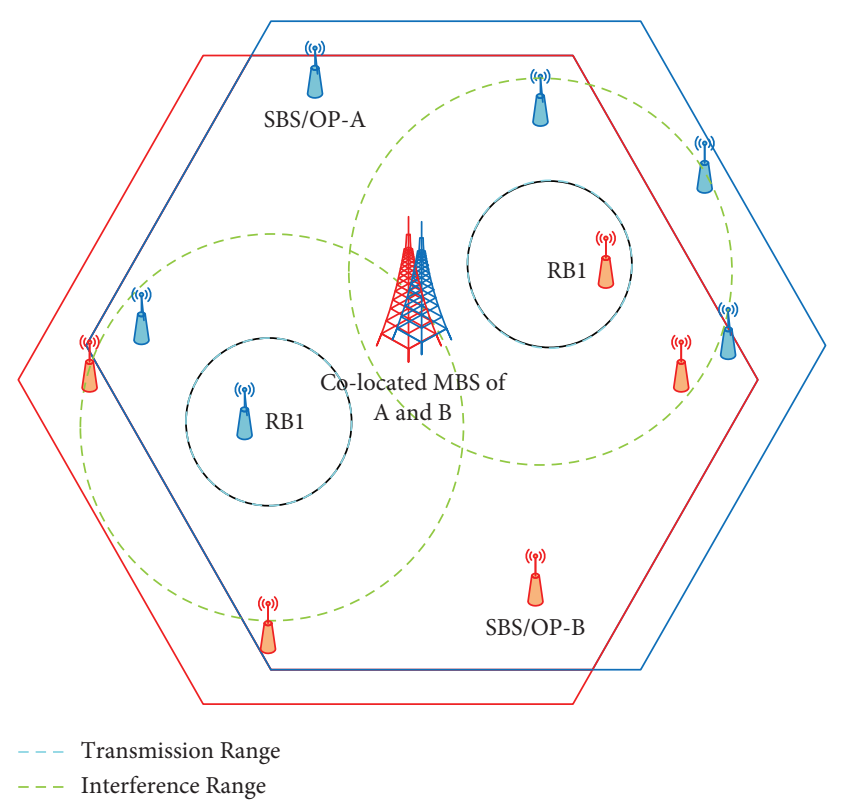

FIGURE 3: Resource block allocation with interference range.

is being used within the green dashed line disk, then it cannot be reused in its interference range. Figure 3 shows the RB allocation for the co-located MBS in HetNets. A small cell user associated with an SBS of the operator $A$ experiences interference from all other SBSs of operators $A$ and $B$, if they are reusing the same $\mathrm{RB}$.

The interference range of a small cell $m$ is represented by $v_{m}$, whereas the MBS are colocated; therefore, each region will have small cells of both operators present. Thus, during SSP, the central controller keeps updating the interference profiles for each small cell user of both operators. The unserved small cell users of both operators will be served by $K_{T}$ resource blocks in SSP, where

$$
K_{T}=\left\{K_{S}\right\} \cup\left\{K_{o}\right\} \text {. }
$$

Algorithm 2 ensures that the same RB is not assigned to any other user within the transmission range or the corresponding interference range. Central controller is aware of the information on how SSP is used, i.e., whether each user is served by operator $A$ or operator $B$. Moreover, it knows about the user connectivity with the specific SBS, the location of SBS, and the set of RBs that form the shared spectrum pool within a specific interference range. Before negotiating a round of allocation, the central controller also knows the radii of transmission and interference range, the interference protocol is modeled by the central controller as both operators exchange their basic information with mutual coordination to a central entity i.e., the central controller. By knowing about all SBSs of both MNOs in each 
RBIR, the small cell base stations know about all interfering units for the scheduled users.

Following are the steps of Algorithm 2.

(i) Step 2, the unserved users are checked by following Algorithm 1 and stored in $\mathbf{Q}_{\mathrm{O}, \mathrm{N}^{\prime \prime}}^{\mathrm{m}}$.

(ii) Step 3, both operators coordinate via central controller to form pool and exchange basic information, i.e., load index and willingness.

(iii) Steps 4-6, load parity is calculated by central controller and depending upon the load parity both operator agree to form SSP of $K_{T}$ resource blocks.

(iv) Step 7, for each unsatisfied SCN user from operator $A$ and SCN user from operator $B$, the interference profile is updated by central controller.

(v) Finally in step 8, the RBs from SSP are allocated to the unsatisfied users of operator $A$. As the SSP is being used by both operators, the resource blocks from SSP are allocated to the users of both operators.

Resource allocation is performed by keeping in view the previous allocation. After allocating RB to each user, every RBIR will generate a new set of interfering units, and then a new user will be assigned to RBs accordingly. In this way, the proposed algorithm ensures that additional interferers, whether interoperator or intra-operator, do not get effected significantly.

4.2. Load-Based Leased Spectrum Pool (LLSP). In this scenario, one of the MNOs has additional resources left after fulfilling its own resource requirement. This MNO (lessor) leases its additional spectrum to the other MNO (lessee) whose resource requirement exceeds its available resources. The major advantage in this case is that no interference is caused, as the leased resources are exclusively used by the lessee MNO for the duration allowed. It is assumed that the overall network load condition does not change significantly for the duration of lease. This is a fair assumption, as in practice, the network load does not change significantly over a few seconds or even minutes.

In LLSP, the $K_{T}$ RBs are used only by operator $O$. Leased spectrum pool with RBIR reduces the interference to a minimum level as the lessor is no more using the RBs from $K_{T}$, and is referred to as the leased spectrum pool. Leasing spectrum may effect an operator's own spectrum usage, but this is managed by ensuring load parity between the lessor and lessees. We assumed here, an operator $O^{\prime}$ will lease its spectrum only in the condition when it has a sufficient amount of free RBs available even after leasing.

In Algorithm 3 with LLSP, the small cell users of operator $O^{\prime}$ no longer cause interference. Therefore, each interference range $\mathscr{M}$ has small cells of operator $O$, i.e.,

$$
\mathscr{M} \leftarrow M_{O}
$$

Since during the LSP the central controller keeps updating the interference profiles for each small cell user; therefore, the unserved small cell users are served by $K_{T}$ RBs in LSP.

The main steps of Algorithm 3 are as follows;

(i) In step 2, the unserved users are checked by following algorithm 1 and stored in $\mathbf{Q}_{\mathrm{O}, \mathrm{N}^{\prime \prime}}^{\mathrm{m}}$.

(ii) Step 3, both operators coordinate via central controller to form pool and exchange basic information, i.e., load index and willingness.

(iii) Steps 4-6, from the information of load index exchanged in step 3, load parity is calculated by central controller and both operator agree to form LSP of $K_{T}$ RBs if the load parity is within the upper limit.

(iv) Step 7, for each unsatisfied SCN user from operator $A$, the interference profile is updated by the central controller.

(v) Finally in step 8, the resource blocks from LSP are allocated to the unsatisfied users of operator $A$. As the LSP is being used by operator $A$ exclusively, the $\mathrm{RBs}$ from LSP are allocated to the users of operator A.

The obvious advantage of spectrum leasing is the elimination of additional interference. In this case, (3) gets modified for both operators as the factor $I_{O^{\prime}, n^{\prime \prime}, k}$ vanishes, which in turn improves the SINR. In the next section, we analyze the scheme that gives an improvement in spectrum trading.

4.3. Complexity Analysis. In this work, heuristic approaches are proposed for RB allocation. Algorithm 1 is a basic greedy way of allocating RBs and its complexity is of the order of $O\left(N_{0}^{\prime \prime} \times K_{T}\right)$. For algorithm 2 , a shared pool is created whose complexity is of the order of $O\left(N_{o}^{\prime \prime} \times K_{T} \times M_{A} \times M_{B}\right)$. Similarly, for algorithm 3 , the complexity is of the order $O\left(N_{o}^{\prime \prime} \times K_{T} \times M_{o}\right)$. Note that $K_{T}$ for algorithms 2 and 3 are different.

\section{Performance Evaluation and Discussion}

In this section, we evaluate the performance of LSSP and LLSP. We consider two operators with co-located MBSs in an HetNet that consists of densely deployed outdoor small cells. An exclusive slice of the spectrum is allocated to small cells and macrocells. A comparison of the proposed trading scheme is performed with the case of no trading and trading with the interference management technique. In Ref. [24], the authors consider the orthogonal and nonorthogonal sharing schemes between two operators. However, we consider the same scenarios, i.e., orthogonal (referred to as leasing) and nonorthogonal (referred to as sharing) for two MNOs with RBIR. A comparison of both cases of LBST in two MNOs with no sharing is also presented. The network layout of the simulation scenario is shown in Figure 4 . The cell site area is divided into circular zones with a BS transmission range of radius $r=250 \mathrm{~m}$. The radius of the interference range is $2 r$. The small cells are static and 
(1) initialize $\mathscr{C}_{\text {o }}$

(2) From Algorithm 1 check $\mathbf{Q}_{\mathrm{O}, \mathrm{N}^{\prime \prime}}^{\mathrm{M}}$ has unsatisfied Users

(3) if $F=1$ operator $O \in\{A, B\}$ coordinate via $\mathscr{C}_{\mathrm{o}}$ exchange load index

(4) Calculate P

(5) while $\mathrm{P}<\delta$ true, both operators agree to form SSP

(6) Operator $O^{\prime}$ share its RBs $K_{S}$ based on sharing factor, SSP has $K_{T}=K_{O} \cup K_{S}$ and update $\mathscr{C}_{\text {o }}$

(7) for $m=1: M_{o}$

(8) $\mathscr{C}_{\mathrm{o}} \leftarrow$ Update interference relationship

(9) for $q=1$ : $\operatorname{size}\left(\mathbf{Q}_{\mathrm{O}, \mathrm{N}^{\prime \prime}}^{\mathrm{m}}\right)$ Start allocating RBs from SSP by avoiding the interference range

(10) end

(11) end

(12) Repeat step 7-10 until $\mathbf{Q}_{\mathrm{O}, \mathrm{N}^{\prime \prime}}^{\mathrm{M}}=$

(13) else

(14) $F=0$

(15) end

Algorithm 2: Co-channel RB allocation of LSSP with RBIR.

(1) initialize $\mathscr{C}_{\mathrm{o}}$

(2) From Algorithm 1 check $\mathbf{Q}_{\mathrm{O}, \mathrm{N}^{\prime \prime}}^{\mathrm{M}}$ has unsatisfied Users

(3) if $F=1$ operator $O \in\{A, B\}$ coordinate via $\mathscr{C}_{0}$ exchange load index

(4) Calculate $P$

(5) while $\mathrm{P}>\delta$, both operators agree to form LSP

(6) Operator $O^{\prime}$ lease its free RBs $K_{L}$, LSP has $K_{T}=K_{O}-K_{L}$ and update $\mathbb{C}_{\mathrm{o}}$

(7) for $m=1: M_{o}$

(8) $\mathscr{C}_{\mathrm{o}} \leftarrow$ Update interference relationship

(9) for $q=1$ : size $\left(\mathbf{Q}_{\mathrm{O}, \mathrm{N}^{\prime \prime}}^{\mathrm{m}}\right)$ Start allocating $\mathrm{RBs}$ from LSP

(10) end

(11) end

(12) Repeat steps 7-10 until $\mathbf{Q}_{\mathrm{O}, \mathrm{N}^{\prime \prime}}^{\mathrm{m}}=$

(13) else

(14) $F=0$

(15) end

Algorithm 3: Co-channel RB allocation of LLSP with RBIR.

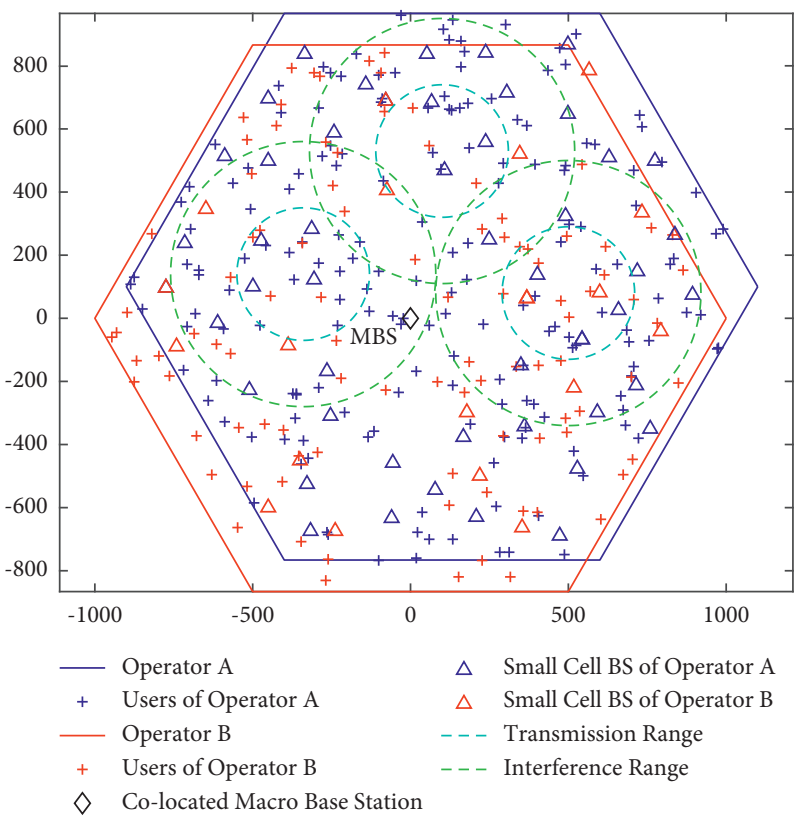

FIGURE 4: Simulated layout of two operator heterogeneous networks. 
TABLE 2: Simulation parameters.

\begin{tabular}{lc}
\hline Parameters & Values \\
Carrier frequency & $2.1 \mathrm{GHz}$ \\
Load on each MNO & $L_{A}=100 \mathrm{users}, L_{B}=50 \mathrm{users}$ \\
System bandwidth & $20 \mathrm{MHz}$ \\
Small cell network BW & $10 \mathrm{MHz}$ \\
Macrocell network BW & $10 \mathrm{MHz}$ \\
No. of RBs for small cell network & 50 \\
No. of RBs for macrocell network & 50 \\
No. of small cell BS for each MNO & $M_{A}=50, M_{B}=20$ \\
Macro BS transmit power & $46 \mathrm{dBm}$ \\
Small cell BS transmit power & $23 \mathrm{dBm}$ \\
Macrocell radius & $1000 \mathrm{~m}$ \\
Small cell radius & $80 \mathrm{~m}$ \\
Load disparity threshold & $\delta$ \\
Noise power & $-174 \mathrm{dBm}$ \\
Channel model & Rayleigh fading channel \\
Path-loss model & $140.1+37.6$ log $(d)$ SBS to user \\
Bandwidth of resource blocks & $128.1+36.7$ log $(d) \mathrm{MBS}$ to user \\
\hline
\end{tabular}

randomly deployed. The small cells of both operators may overlap.

The users are distributed randomly according to a uniform distribution. Each SBS serves 5-6 users. Prior to using LBST, i.e., when neither LSSP nor LLSP are used, the simulation is performed without the RBIR. The resources from the shared or leased spectrum pools are allocated by the following RBIR to avoid any additional interoperator interference. The radius of the transmission range is kept fixed at $250 \mathrm{~m}$. To improve the cell edge performance, relatively more small cells are deployed at the macrocell edges as the users closer to the MBS are likely to experience high SINR from it. The users that are not present in the close proximity of MBS are associated with those SBSs that offer the highest SINR. The simulation parameters are summarized in Table 2.

Figure 5 demonstrates the effect of radii of interference range on the average data rate per user of both operators for LSSP and LLSP. With the increase in the radius of interference range, the average data rate of users is decreased. This happens because by increasing the radius of interference range, the interference experienced by users also increases. When the interference range is less, then sharing (LSSP) results in higher average data rate per user for both operators. However, when the interference range is increased beyond $300 \mathrm{~m}$ and $320 \mathrm{~m}$ for operators B and A, respectively, the leasing (LLSP) results in a higher average data rate. This trend is intuitive, as with increasing interference, and sharing further adds to the interference which in turn adversely effects the end user data rates. When the interference range increases, the maximum transmit power increases and not the assigned power; therefore, the transmission range remains the same.

Figure 6 shows the throughput comparison of LSSP and LLSP that are compared with a baseline scheme where no spectrum trading is performed, i.e., without LBST (neither LSSP nor LLSP). In this case, we consider the load disparity to be greater than the threshold, i.e., $\mathrm{P}>\delta$, and the operator

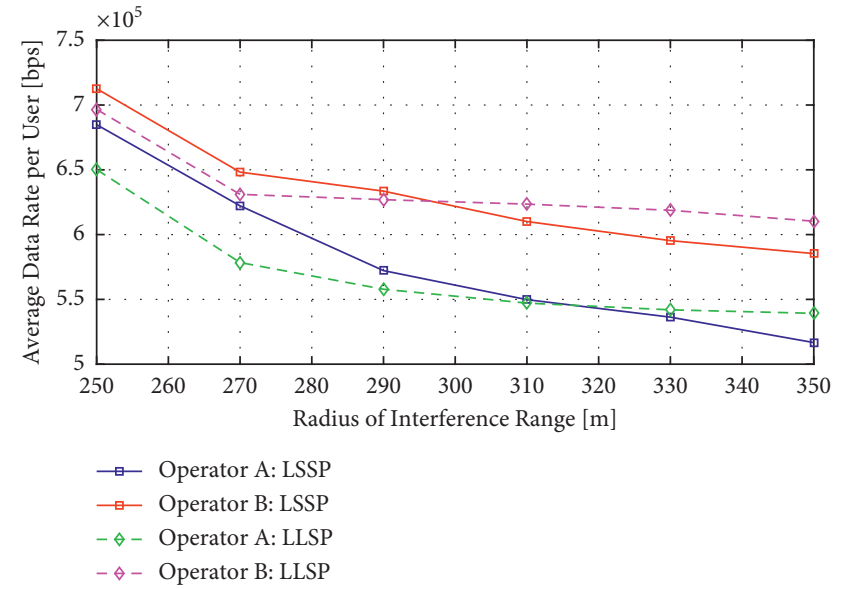

Figure 5: Average data rate per user with variable radii of interference range for operators $\mathrm{A}$ and $\mathrm{B}$.

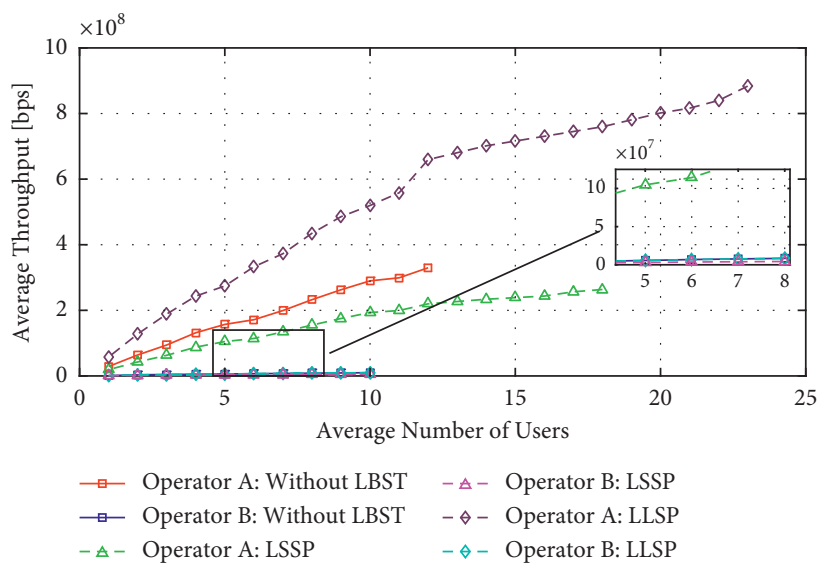

FIgURE 6: Performance comparison of operator A and B without LBST, with LSSP, and with LLSP. 


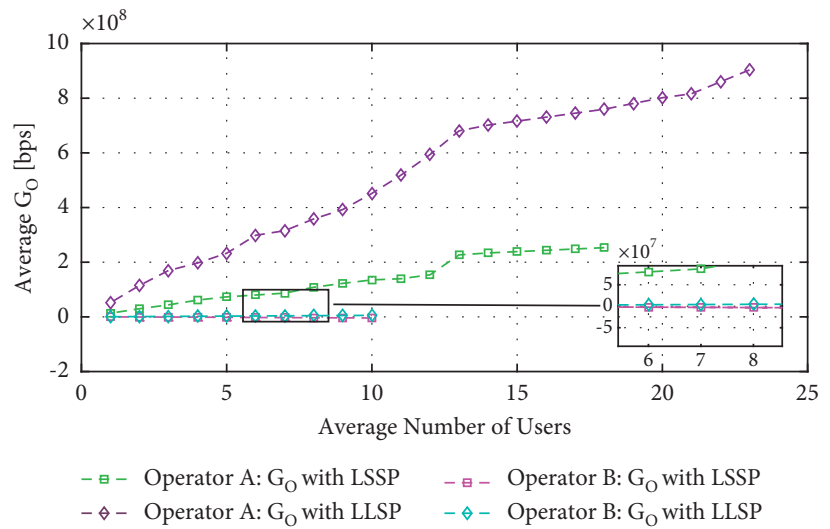

Figure 7: Average throughput gain achieved by operator A and operator B with LSSP and LLSP.

$A$ is significantly over loaded (more users or data intensive requirement of the users) compared to operator $B$. For the case of operator $A$, a significant increase in the performance of LLSP is observed, whereas a decrease in the performance of LSSP is observed compared to LBST. This is due to the presence of load disparity between the two operators $L_{B}>\delta L_{A}$, and in this situation leasing is the preferred approach. Similarly, Operator $B$ sacrifices its per user throughput as it experiences additional interference from operator $A$. However, in the case of LLSP, the operator $A$ receives a favor in the form of increased per user throughput as well as the number of satisfied users. Moreover, the overlapping curves of Algorithm 1 and LLSP for operator $B$ indicate that the performance of operator $B$ remains unaffected. This is primarily due to operator $B$ being significantly less loaded compared to operator $A$. A gain in average throughput is observed for both operators compared to the case of no spectrum trading. From this, we can conclude that for load disparate scenarios, spectrum leasing is more beneficial and may be adopted by the MNOs.

The gain in net throughput, i.e., $G_{O}$ from (7), achieved by both operators is shown in Figure 7. For the operator $A$, both algorithms result in an increase in the overall network throughput. Especially, LLSP gives the highest gain in throughput, much more than LSSP. As explained earlier, this happens because the operator $A$ is granted additional spectrum for exclusive use. Whereas in the case of LSSP, the operator $A$ shares the additional spectrum with operator $B$, which results in a relatively lesser increase in network throughput due to interference from shared resources. The overall gain in network throughput for the operator $A$ increases almost proportionately with the increase in the average number of users. For operator $B$, there is no gain in throughput for either LLSP or LSSP. For operator $B$, the average number of users is much less, i.e., 10, which indicates a relatively lesser traffic load for operator $B$.

Figure 8 shows the effect of sharing/leasing factor (percentage of resources shared/leased) on each MNO's average throughput. The curve shows that while the sharing/ leasing factor is varied from 0 to $100 \%$, the average throughput of operator $A$ is increased for both algorithms. For operator $B$, there is no difference in the average

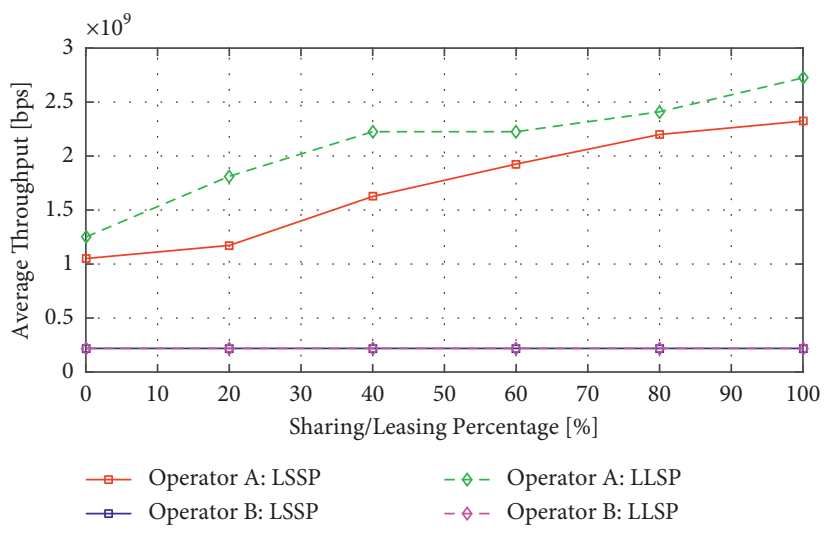

FIgURE 8: Relationship of average throughput with different percentage of sharing/leasing factor for LSSP and LLSP.

throughput as it shares/leases its resources after satisfying its own throughput requirement for a lower traffic load.

Figure 9 shows the performance of both the operators with and without LBST in terms of the percentage of user satisfaction. A user is satisfied if a minimum quality of service (QoS) requirement is satisfied. The percentage of user satisfaction is expressed as

$$
\mathbb{N}=\frac{\sum_{i=1}^{N_{O}^{\prime}} e_{i}}{N_{O}^{\prime \prime}} \times 100,
$$

where $e_{i}=1$ if user is satisfied, otherwise it is 0 . It is obvious that the number of satisfied users of the operator $A$ while using LLSP and LSSP are greater compared to LBST. LLSP gives the best performance and it results in around 15\% greater user satisfaction for operator $A$ compared to LSSP. The performance of the collaborator, i.e., operator $B$, remains unaffected for both algorithms, as its users are already satisfied due to lesser traffic load and are well served with the available resources.

From the results, we conclude that (a) for small cells with small transmission range or interference range, spectrum trading should be preferred, (b) for load disparate scenarios, spectrum leasing should be the preferred choice, and (c) 


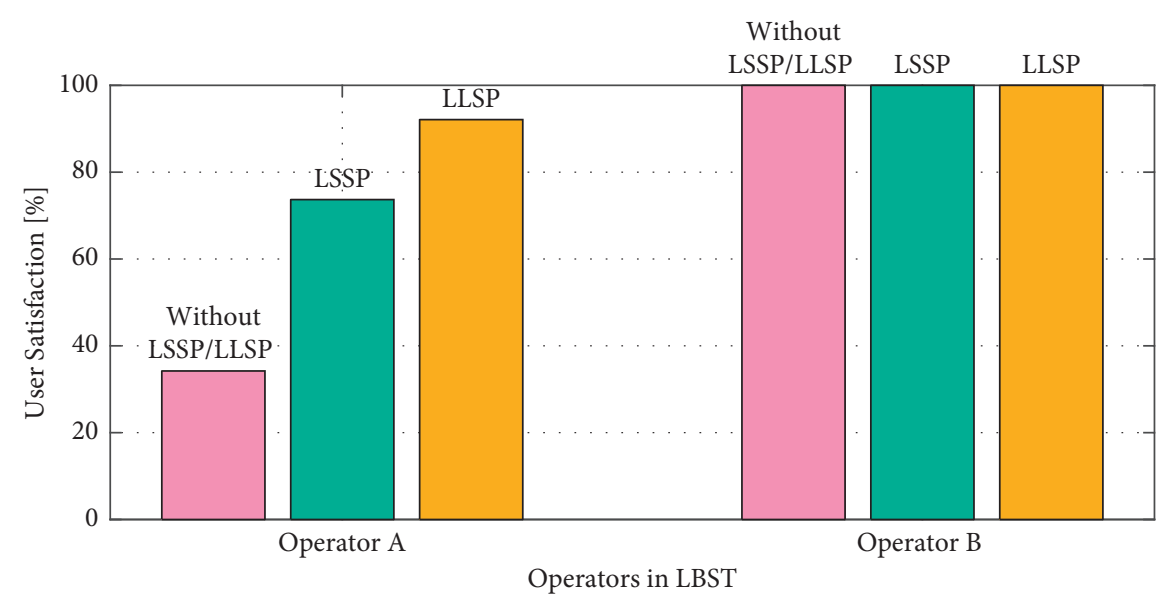

FIGURE 9: User satisfaction of operator $A$ and $B$ without LSSP/LLSP, with LSSP, and with LLSP.

spectrum trading provides an increase in throughput gain compared to nontrading schemes. The conclusions are general and applicable in all standard scenarios. However, the interference range or the difference of load disparity value might depend on the channel conditions.

\section{Conclusion and Future Work}

In this paper, we have proposed and analysed interoperator spectrum trading for outdoor small cells of two MNOs with disparate traffic loads. While doing cooperative spectrum trading on the resource block level, the interference is managed to an acceptable limit by using resource block allocation with interference range. We have analysed two main approaches, i.e., spectrum sharing (LSSP) and spectrum leasing (LLSP), which are dependent on load traffic and ensure that the user receives the highest SINR from the corresponding SBS in each scenario. In addition to that, the performance of the operators for each approach is evaluated for disparate load traffic while considering the pursued constraints, i.e., network throughput, user satisfaction percentage, and average user data rate. Based on that, the following observations have been drawn: when the interference is less, as represented by the radius of interference range, spectrum sharing results in a higher average data rate; however, when the interference increases, leasing is more advantageous in delivering higher data rates. In terms of average throughput and user satisfaction percentage, spectrum sharing and leasing both resulted in increased performance of the operator without affecting the performance of the collaborators.

In the future, it is planned to study the analytical model of spectrum trading as a function of load disparity. This model can be extended for multiple MNOs with static and mobile users. Spectrum trading for the case of noncolocated MNOs can be an interesting problem to explore.

\section{Data Availability}

Data are available on request from Hafiza Ammara Khurshid, ammara09es24@gmail.com.

\section{Conflicts of Interest}

The authors declare that they have no conflicts of interest.

\section{References}

[1] M. U. Iqbal, E. A. Ansari, and S. Akhtar, "Interference mitigation in HetNets to improve the QoS using Q-learning," IEEE Access, vol. 9, pp. 32405-32424, 2021.

[2] V. J. Kotagi, P. K. Thiruvasagam, and C. S. R. Murthy, "Learn to breathe: an energy efficient inter-operator proximal spectrum sharing," IEEE Transactions on Green Communications and Networking, vol. 4, no. 3, p. 1, 2020.

[3] J. Zhang and G. De la Roche, Femtocells: Technologies and Deployment, Wiley Online Library, Hoboken, NJ, USA, 2010.

[4] T. Frisanco, P. Tafertshofer, P. Lurin, and R. Ang, "Infrastructure sharing and shared operations for mobile network operators from a deployment and operations view," IEEE Network Operations and Management Symposium, vol. 1, pp. 129-136, 2008.

[5] J. Kibilda, P. D. Francesco, F. Malandrino, and L. A. DaSilva, "Infrastructure and spectrum sharing trade-offs in mobile networks," in Proceedings of the IEEE International Symposium on Dynamic Spectrum Access Networks, pp. 348-357, Stockholm, Sweden, September 2015.

[6] L. Anchora, L. Badia, E. Karipidis, and M. Zorzi, "Capacity gains due to orthogonal spectrum sharing in multi-operator lte cellular networks," in Proceedings of the International Symposium on Wireless Communication Systems, pp. 286290, Paris, France, August 2012.

[7] E. Jorswieck, L. Badia, T. Fahldieck et al., "Resource sharing improves the network efficiency for network operators," in Wireless World Research Forumvol. 52, no. 3, 2011.

[8] F. Mazzenga, M. Petracca, R. Pomposini, F. Vatalaro, and R. Giuliano, "Performance evaluation of spectrum sharing algorithms in single and multi operator scenarios," in Proceedings of the IEEE Vehicular Technology Conference, pp. 1-5, Budapest, Hungary, May 2011.

[9] L. Anchora, M. Mezzavilla, L. Badia, and M. Zorzi, “A performance evaluation tool for spectrum sharing in multi-operator lte networks," Computer Communications, vol. 35, no. 18 , pp. 2218-2226, 2012.

[10] A. Osseiran, F. Boccardi, V. Braun et al., "Scenarios for $5 g$ mobile and wireless communications: the vision of the metis 
project," IEEE Communications Magazine, vol. 52, no. 5, pp. 26-35, 2014.

[11] C. Yang, J. Li, M. Guizani, A. Anpalagan, and M. Elkashlan, "Advanced spectrum sharing in $5 \mathrm{~g}$ cognitive heterogeneous networks," IEEE Wireless Communications, vol. 23, no. 2, pp. 94-101, 2016.

[12] S. Farhat, A. E. Samhat, S. Lahoud, and B. Cousin, "Pricing strategies in multi-operator heterogeneous wireless networks," in Proceedings of the 2015 7th International Conference on New Technologies, Mobility and Security (NTMS), pp. 1-5, Paris, France, July 2015.

[13] M. Rahman, M. Yuksel, and T. Quint, "Regulating freeriding in inter-operator spectrum sharing," in Proceedings of the 2018 IEEE International Symposium on Dynamic Spectrum Access Networks (DySPAN), pp. 1-10, Seoul, Korea, October 2018.

[14] M. Rahman, M. Yuksel, and W. Saad, "Regulations and strategies in subsidy-based spectrum sharing markets," in Proceedings of the 2019 IEEE International Symposium on Dynamic Spectrum Access Networks (DySPAN), pp. 1-10, Newark, NJ, USA, November 2019.

[15] S. Zheng, T. Han, Y. Jiang, and X. Ge, "Smart contract-based spectrum sharing transactions for multi-operators wireless communication networks," IEEE Access, vol. 8, pp. 8854788557, 2020.

[16] A. Asghar, H. Farooq, and A. Imran, "Concurrent cco and lb optimization in emerging hetnets: a novel solution and comparative analysis," in Proceedings of the 2018 IEEE 29th Annual International Symposium on Personal, Indoor and Mobile Radio Communications (PIMRC), pp. 1-6, Bologna, Italy, September 2018.

[17] N. Deng and M. Haenggi, "Sinr and rate meta distributions for hons with joint spectrum allocation and offloading," IEEE Transactions on Communications, vol. 67, no. 5, pp. 37093722, 2019.

[18] R. H. Tehrani, S. Vahid, D. Triantafyllopoulou, H. Lee, and K. Moessner, "Licensed spectrum sharing schemes for mobile operators: a survey and outlook," IEEE Communications Surveys \& Tutorials, vol. 18, no. 4, pp. 2591-2623, 2016.

[19] B. Singh, K. Koufos, and O. Tirkkonen, "Co-primary interoperator spectrum sharing using repeated games," in Proceedings of the IEEE International Conference on Communication Systems, pp. 67-71, London, UK, June 2014.

[20] B. Singh, K. Koufos, O. Tirkkonen, and R. Berry, "Co-primary inter-operator spectrum sharing over a limited spectrum pool using repeated games," in Proceedings of the IEEE International Conference on Communications, pp. 1494-1499, London, UK, June 2015.

[21] B. Singh, K. Koufos, O. Tirkkonen, and R. Jantti, "Repeated spectrum sharing games in multi-operator heterogeneous networks," in Proceedings of the IEEE International Symposium on Dynamic Spectrum Access Networks, pp. 221-228, Stockholm, Sweden, September 2015.

[22] P. Luoto, P. Pirinen, M. Bennis, S. Samarakoon, S. Scott, and M. Latva-aho, "Co-primary multi-operator resource sharing for small cell networks," IEEE Transactions on Wireless Communications, vol. 14, no. 6, pp. 3120-3130, 2015.

[23] P. Luoto, M. Bennis, P. Pirinen, S. Samarakoon, and M. LatvaAho, "Enhanced co-primary spectrum sharing method for multi-operator networks," IEEE Transactions on Mobile Computing, vol. 16, no. 12, pp. 3347-3360, 2017.

[24] E. Jorswieck, L. Badia, T. Fahldieck, E. Karipidis, and J. Luo, "Spectrum sharing improves the network efficiency for cellular operators," IEEE Communications Magazine, vol. 52, no. 3, pp. 129-136, 2014.
[25] T. Sanguanpuak, S. Guruacharya, N. Rajatheva, M. Bennis, D. Niyato, and M. Latva-Aho, "Multi-operator spectrum sharing using matching game in small cells network," in Proceedings of the IEEE International Conference on Communications, pp. 1-6, Kuala Lumpur, Malaysia, May 2016.

[26] M. G. Kibria, G. P. Villardi, K. Nguyen, K. Ishizu, and F. Kojima, "Heterogeneous networks in shared spectrum access communications," IEEE Journal on Selected Areas in Communications, vol. 35, no. 1, pp. 145-158, 2017.

[27] J. Kibilda, N. J. Kaminski, and L. A. DaSilva, "Radio access network and spectrum sharing in mobile networks: a stochastic geometry perspective," IEEE Transactions on Wireless Communications, vol. 16, no. 4, pp. 2562-2575, 2017.

[28] R. Gangula, D. Gesbert, J. Lindblom, and E. G. Larsson, “On the value of spectrum sharing among operators in multicell networks," in Proceedings of the IEEE 77th Vehicular Technology Conference, pp. 1-5, Dresden, Germany, June 2013.

[29] S. Anand, S. Sengupta, and R. Chandramouli, "Price-bandwidth dynamics for WSPs in heterogeneous wireless networks," Physical Communication, vol. 12, pp. 63-78, 2014.

[30] R. K. Saha, "Realization of licensed/unlicensed spectrum sharing using eicic in indoor small cells for high spectral and energy efficiencies of $5 \mathrm{~g}$ networks," Energies, vol. 12, no. 14, pp. 1-28, 2019.

[31] R. K. Saha, "On exploiting millimeter-wave spectrum trading in countrywide mobile network operators for high spectral and energy efficiencies in 5g/6g era," Sensors, vol. 20, no. 12, 2020.

[32] H. S. Ghadikolaei, H. Ghauch, G. Fodor, M. Skoglund, and C. Fischione, "A hybrid model-based and data-driven approach to spectrum sharing in mmwave cellular networks," IEEE Transactions on Cognitive Communications and Networking, vol. 6, no. 4, pp. 1269-1282, 2020.

[33] A. AlAmmouri, H. ElSawy, and M. S. Alouini, "Load-aware modeling for uplink cellular networks in a multi-channel environment," in Proceedings of the 2014 IEEE 25th Annual International Symposium on Personal, Indoor, and Mobile Radio Communication (PIMRC), pp. 1591-1596, Washington, DC, USA, September 2014.

[34] J. Lorincz, T. Garma, and G. Petrovic, "Measurements and modelling of base station power consumption under real traffic loads," Sensors, vol. 12, no. 4, pp. 4281-4310, 2012.

[35] U. Paul, A. P. Subramanian, M. M. Buddhikot, and S. R. Das, "Understanding traffic dynamics in cellular data networks," in Proceedings of the IEEE INFOCOM, pp. 882-890, Shanghai, China, April 2011.

[36] P. Cardieri, "Modeling interference in wireless ad hoc networks," IEEE Communications Surveys \& Tutorials, vol. 12, no. 4, pp. 551-572, 2010.

[37] F. Z. Kaddour, M. Pischella, P. Martins, E. Vivier, and L. Mroueh, "Opportunistic and efficient resource block allocation algorithms for lte uplink networks," in Proceedings of the Wireless Communications and Networking Conference, pp. 487-492, IEEE, Shanghai, China, April 2013.

[38] R. A. Kalden, Mobile internet traffic measurement and modeling based on data from commercial GPRS networks, $\mathrm{PhD}$ Thesis, University of Twente Research Information Logo, Enschede, Netherlands, 2004. 